\title{
Inducing involuntary and voluntary mental time travel using a laboratory paradigm
}

\author{
Scott N. Cole ${ }^{1,2} \cdot$ Søren R. Staugaard ${ }^{1} \cdot$ Dorthe Berntsen $^{1}$
}

Published online: 21 October 2015

(C) Psychonomic Society, Inc. 2015

\begin{abstract}
Although involuntary past and future mental time travel (MTT) has been examined outside the laboratory in diary studies, MTT has primarily been studied in the context of laboratory studies using voluntary construction tasks. In this study, we adapted and extended a paradigm previously used to elicit involuntary and voluntary memories (Schlagman \& Kvavilashvili in Memory \& Cognition, 36, 920-932, 2008). Our aim was - for the first time - to examine involuntary and voluntary future MTT under controlled laboratory conditions. The involuntary task involved a monotonous task that included potential cues for involuntary MTT. Temporal direction was manipulated between participants whereas retrieval mode was manipulated within participants. We replicated robust past-future differences, such as the future positivity bias. Additionally, we replicated key voluntary-involuntary differences: Involuntary future representations had similar characteristics as involuntary memories in that they were elicited faster, were more specific, and garnered more emotional impact than their voluntary counterparts. We also found that the future and past involuntary MTT led to both positive and negative mood impact, and that the valence of the impact was associated with the emotional valence of the event. This study advances scientific understanding of involuntary future representations in healthy populations and validates a laboratory paradigm that can be flexibly and systematically utilized to explore different characteristics of voluntary and
\end{abstract}

Scott N. Cole

cole.s.n80@gmail.com

1 Center on Autobiographical Memory, Aarhus University, Aarhus, Denmark

2 Department of Psychology, York St. John University, York YO31 7EX, UK involuntary MTT, which has not been possible within naturalistic paradigms.

Keywords Autobiographical memory $\cdot$ Involuntary memory . Episodic future thinking $\cdot$ Memory $\cdot$ Mental time travel

Each day, people can be heard talking about their realized past and possible future; from footballers who talk about past glories and future challenges, to stockbrokers who talk about that lost opportunity and that future windfall. Over the past decade, the shared neuro-cognitive processes involved in episodic past and future thinking have been the focus of intense research (see Schacter et al., 2012, for a review). This capacity - to withdraw from the present reality and mentally simulate a past or future event as if one was there - has been termed mental time travel (MTT; see Tulving, 2002; Wheeler, Stuss, \& Tulving, 1997). It is argued that MTT into the past and future relies primarily upon the episodic memory system, which can be distinguished from the ability to store and retrieve semantic information (see Schacter \& Addis, 2007; Berntsen \& Bohn, 2010; Klein, 2013).

However, little is known about those past or future thoughts that come to mind unbidden, without willful generation processes (despite a long history of automatic and controlled processes in memory research; e.g., see B. A. Anderson et al., 2011; Hintzman, 2011; Kelley \& Jacoby, 2000; SteMarie \& Jacoby, 1993). Although several studies have induced involuntary autobiographical memories using laboratory techniques (Ball, 2007; Berntsen, Staugaard, \& Sørensen, 2013; Schlagman \& Kvavilashvili, 2008; Uzer, Lee, \& Brown, 2012), to our knowledge, this is the first study to use a laboratory paradigm to examine past and future events that are retrieved voluntarily and involuntarily. In so doing, we attempt to fulfil two aims: (1) to validate a new laboratory 
method of involuntary MTT and (2) to understand the emotional, representational, and cognitive characteristics of these four mental representation types, especially the phenomenon of involuntary future MTT, which has hitherto been studied using naturalistic methods. With this paradigm, we aim to simulate the key cuing conditions of naturally occurring MTT in a controlled laboratory setting. In addition to answering questions that require a controlled laboratory approach, one key question is whether we can replicate results from previous naturalistic studies using this paradigm, thus providing external validity.

\section{Studies of voluntary and involuntary memories}

To date, much of autobiographical memory research has investigated memories retrieved voluntarily. To recall a specific autobiographical memory, a generative retrieval process is initiated, which is iterative and follows a cycle of top-down processes involving search, evaluation, and elaboration (see Conway, 2005). In autobiographical memory (and MTT research generally), the cue word task (Crovitz \& Schiffman, 1974) is commonly used, which involves participants attempting to recall a personal past event associated with a cue word (e.g., tree).

The majority of studies on involuntary autobiographical memory adopt a structured diary method (e.g., Berntsen, 1996, 1998; Berntsen \& Hall, 2004; Kvavilashvili \& Mandler, 2004; Mace, 2004). Diary studies have shown that cues are identified by participants in around $80 \%$ to $90 \%$ of involuntary memories (Berntsen, 1996; Berntsen \& Hall, 2004; Kvavilashvili \& Mandler, 2004; Mace, 2004) and that involuntary memories generally occur when attention is diffuse, such as during rest and routine tasks (e.g., Berntsen, 1998; Kvavilashvili \& Mandler, 2004).

It has been argued that although voluntary and involuntary memories rely on the same episodic system (Berntsen, 2010), they differ in the way they are retrieved, hence, why differences between the two are largely attributable to differences in retrieval mode rather than differences in encoding and maintenance (Berntsen, 2010). One reliable finding is that people produce a higher proportion of specific involuntary memories compared with voluntarily retrieved memories (e.g., Berntsen, 1998; Berntsen \& Hall, 2004; Berntsen \& Jacobsen, 2008; Mace, 2006). Involuntary memories tend to impact one's current mood to a greater extent than their voluntary counterparts (see Berntsen, 2009, for a review), which may be due to their unplanned occurrence, leaving little room for emotion regulation (Berntsen, 2010). In addition, involuntary memories are elicited faster than voluntary memories (Berntsen et al., 2013; Schlagman \& Kvavilashvili, 2008), suggesting that their retrieval is less effortful. Convergent results have been found in neuroimaging, such that, compared with involuntary retrieval, voluntary retrieval is associated with greater activity in brain regions implicated in high-level control processes (Hall et al., 2014). Nevertheless, a common set of neural structures associated with recollection are activated for both voluntary and involuntary episodic memories (Hall et al., 2014). Hall and colleagues suggest that although the means by which involuntary and voluntary memories are elicited differs, the resulting episodic representations remain similar (Hall et al., 2014). Finally, involuntary memory cues arise from transient aspects of the present environment or thoughts (e.g., Berntsen, 1998; Mace, 2004), whereas voluntary memories have a more explicit cue (e.g., Conway, 2005).

\section{Past and future episodic thinking and the concept of MTT}

This episodic memory system, and by association MTT, is said to rely on a unified neurocognitive network (see Schacter et al. 2012, for a review). Studies of healthy adults, for example, have demonstrated an overlapping core brain network responsible for MTT into the past and future (Addis, Wong \& Schacter, 2007; Hassabis, Kumaran, \& Maguire, 2007; Szpunar, Watson, \& McDermott, 2007). Nevertheless, there are well-documented differences between re/constructions of past and future events. The most reliable differences being that past events contain a greater level of vividness and sensoryperceptual detail (Berntsen \& Bohn, 2010; D’Argembeau \& Van der Linden, 2004, 2006; Gamboz, Brandimonte, \& de Vito, 2010; Hassabis, Kumaran, \& Maguire, 2007) and more regularly refer to specific events (R. J. Anderson \& Dewhurst, 2009; Berntsen \& Jacobsen, 2008; Miles \& Berntsen, 2011) than future representations. In contrast to memories, future constructions are also moderately novel, containing aspects of previous experience (Gamboz et al., 2010). In terms of emotional content, although both forms of MTT are generally positive when participants self-select events, future representations are reliably rated as more emotionally positive than their mnemonic counterparts (i.e., future positivity bias; see Berntsen \& Jacobsen, 2008; Berntsen \& Bohn, 2010; Rasmussen \& Berntsen, 2013). It is also reported that future MTT is more personally important to the self than past MTT (Addis, Wong \& Schacter, 2008; Berntsen \& Bohn, 2010). Finally, it has been demonstrated that future representations are temporally closer to the present than their mnemonic counterparts (Addis et al., 2008; Spreng \& Levine, 2006; see also Berntsen \& Jacobsen, 2008; Finnbogadóttir \& Berntsen, 2011; Miles \& Berntsen, 2011).

Drawing upon results from neuroimaging (e.g., Addis et al., 2007; Szpunar, et al., 2007) and cognitive neuropsychology (e.g., Berryhill, Picasso, Arnold, Drowos, Olson, 2010), it has been suggested that future imagining is more cognitively effortful than remembering (Anderson \& Dewhurst, 2009; Berntsen \& Bohn, 2010; Cole, Morrison, \& Conway, 2013; Schacter \& Addis, 2007). However, when 
measuring event generation speed, results have proved inconclusive, with some studies demonstrating slower latencies for future events (Miles \& Berntsen, 2011; Anderson, Dewhurst $\&$ Nash, 2012, Experiments 1 and 2, although in Experiment 1 this difference depended upon cue imageability; Rasmussen \& Berntsen, 2014) and some showing no statistically significant difference (Addis et al., 2007, 2009; D'Argembeau \& Van der Linden, 2004; Anderson et al., 2012, Experiment 3). Consequently, it was an open question whether there would be less indications of cognitive effort (measured by latency) associated with past versus future voluntary re/construction.

The first study, examining involuntary MTT into the past and future was conducted by Berntsen and Jacobsen (2008). This study, and one other (Finnbogadóttir \& Berntsen, 2011), employed the diary methodology described above, although in addition to involuntary past events, participants were instructed that future events may also, effortlessly, come to mind. Findings indicated that future projections were as frequent as rememberings of past events (Berntsen \& Jacobsen, 2008; Finnbogadóttir \& Berntsen, 2013, around 20 per day). Notably, involuntary future MTT differed from voluntary future MTT in the same ways as for involuntary memories: Regardless of temporal orientation, involuntary representations were more specific and had greater emotional impact. Additionally, involuntary MTT arose in situations involving diffuse attention and had identifiable triggers either in the external environment or internal thoughts (Berntsen \& Jacobsen, 2008; Finnbogadóttir \& Berntsen, 2011). The differences found between past and future involuntary MTT were consistent with the voluntary MTT literature (see above). Hence, overall, retrieval mode and temporal direction operated independently.

\section{The study design}

In Schlagman and Kvavilashvili's (2008) laboratory paradigm, autobiographical memories were elicited by simulating the conditions in which involuntary memories occur in everyday life. The paradigm involves a monotonous "primary" task, which engenders diffuse attention (similar to daily life tasks; e.g., washing the dishes) while continuously presenting familiar word phrases. Participants must pause the task whenever they experience an involuntary memory. This design uniquely enabled latency data to be recorded, which was determined by calculating the difference between the emergence of the word phrase and the registering of the memory. Voluntary memories were elicited using a similar cue presentation method but without the presence of a concentration task.

We use the paradigm developed by Schlagman and Kvavilashvili (2008) for the following reasons: First, it is a well-established method for measuring involuntary autobiographical memories in a laboratory setting (e.g., Kvavilashvili \& Schlagman, 2011; Schlagman \& Kvavilashvili, 2008;
Schlagman et al., 2009). Second, because the participants are asked to record their involuntary memories as they occur "in real time" during a vigilance task, it relies little on retrospection. This is important in order to avoid contaminating self-report with guessing and personal beliefs (Ericsson \& Simon, 1980; Nisbett \& Wilson, 1977) and also because it is well-known that involuntary memories are rapidly forgotten if not recorded immediately (Berntsen, 1996). Third, this paradigm was developed to resemble the conditions for having involuntary memories in real life, such as their cuedependent nature and the fact that they typically occur during nondemanding tasks. Also, by using this paradigm Schlagman and Kvavilashvili (2008) were able to replicate key findings concerning specificity from the diary studies. For these reasons, we chose to employ the original paradigm developed by Schlagman and Kvavilashvili and extend this paradigm to involuntary future MTT.

Key aims of the present study were to assess whether both past and future autobiographical thoughts could be elicited using this paradigm, and to compare subjective and objective characteristics of laboratory-elicited past and future involuntary MTT. Measuring latency across the four event types also allows exploration of the role of cognitive effort in involuntary and voluntary MTT, as reaction time (RT) is often equated with cognitive effort, with less time spent on tasks indicating greater cognitive ease (see Kahneman, 2011, for a review). Furthermore, unlike Schlagman and Kvavilashvili (2008), we extended our measures to include, among others, emotional impact, emotional intensity, and two measures of self-salience, in order to provide a parallel to measures in naturalistic studies. In sum, this paradigm offers the opportunity to examine and scrutinize the characteristics of involuntary past and future thoughts in a far more controlled way than has been possible in the past.

\section{Hypotheses}

As previous studies of past and future involuntary MTT have shown independent effects of retrieval mode and temporal direction, our hypotheses focus on main effects. Drawing upon reviewed studies, we made several hypotheses regarding expected differences between involuntary and voluntary MTT. Crucially, we predicted that involuntary MTT would more frequently be about specific events and would be elicited faster than voluntary MTT. We also predicted that involuntary MTT would more frequently lead to mood change. Based on an absence of clear differences in the literature (see Berntsen, 2009, for a review), we do not make any predictions regarding the differences between emotional qualities (i.e., emotional valence or emotional intensity) of the voluntarily and involuntarily retrieved events themselves. We would expect, however, that the valence of the representation would be congruent with mood impact (as found by Berntsen, 1996). 
Based on previous studies on MTT, we predicted the following differences between past and future representations: Future MTT was predicted to involve less sensoryperceptual vividness, be less specific, more emotionally positive, more important to the self (conceptualized here as importance to life story and identity; see Berntsen \& Bohn, 2010) and dated nearer the present than its mnemonic counterparts. According to previous studies (e.g., Addis et al., 2007, 2009; Anderson et al., 2012; D'Argembeau \& Van der Linden, 2004; Miles \& Berntsen, 2011; Rasmussen \& Berntsen, 2014), we had no firm predictions whether voluntary past events would be faster to generate than voluntary future ones. Due to these inconclusive results concerning voluntary MTT and the lack of previous data on the retrieval dynamics of involuntary future representations, an interaction for the latency variable was not predicted. In relation to previous research, we also expected future thoughts, whether voluntary or involuntary, to be moderately novel, including elements of previous experience (Gamboz et al., 2010).

\section{Method}

\section{Participants}

Participants were recruited from a database of volunteers at the Center on Autobiographical Memory Research and advertisements at Aarhus University, with the stated necessity that participants must speak Danish. A total number of 64 participants completed the experiment (32 for each temporal direction condition), each receiving two cinema tickets for compensation. Four participants were excluded from the past condition analyses due to noncompliance with concentration task instructions ( $N=1$; e.g., confusion over button press to identify targets), an absence of any involuntary memories $(N=1)$, and self-reported mental illness $(N=2)$, and five were excluded from the future condition analyses due to noncompliance with concentration task instructions $(N=4)$ and reporting only involuntary memories or images not concerning the future $(N$ $=1$ ). The remaining participants who were included in analyses reported at least one representation in both retrieval mode conditions (past, $N=28$; future, $N=27$ ), were neurologically and psychologically healthy, and were comparable in terms of age (past: $M=24.29, S D=6.19$; future: $M=24.33, S D=6.93$ ) and male:female ratio (past $=5: 23$; future $=7: 20$ ).

\section{Design}

A mixed factorial $2 \times 2$ design was employed. The betweengroup factor was temporal direction (past, future) and the within-group factor was retrieval mode (involuntary, voluntary). Participants were deliberately given misinformation that the "primary" aim in the involuntary condition was to detect infrequently presented targets (vertical lines) among nontargets (horizontal lines). Their other task was to report involuntary representations. In order to conceal the main purpose of the involuntary condition (i.e., memory or future MTT), this condition always preceded the voluntary condition, consistent with previous work (e.g., Schlagman \& Kvavilashvili, 2008).

\section{Materials}

Involuntary and voluntary conditions were both presented on E-Prime Professional (Version 2.0) on desktop computers. The stimuli consisted of cue phrases (e.g., coffee jar, lucky find) embedded within black line arrays that were distributed on the white background of each slide. All phrases were presented in 18-point Arial font. The phrases were presented centrally on the screen. Line arrays varied randomly in the amount of lines (4-8 variations) and how they were arranged on the screen (12 variations) but were either horizontal or vertical. Each slide was presented for $1.5 \mathrm{~s}$ (see Fig. 1 for an illustrative diagram). These experimental details were consistent with Schlagman and Kvavilashvili (2008). The involuntary condition consisted of 600 word phrases (extracted from the involuntary cue phrases from Schlagman \& Kvavilashvili, 2008) and was presented to participants as a vigilance task in which the primary task required the identification of targets. Targets were vertical arrays $(N=11)$ that occurred infrequently throughout the task (every 40-60 trials); all other arrays were horizontal (589). Cue phrases were translated from an English cue phrases list (used in Schlagman \& Kvavilashvili, 2008). The voluntary cue phrases were a subset of 12 cue phrases selected from 24 cue phrases used in the voluntary condition in Schlagman and Kvavilashvili (2008). Voluntary cue phrases were not presented in the involuntary condition and vice versa. Cues in involuntary and voluntary conditions did not differ in terms of cue valence and, as shown in Schlagman and Kvavilashvili, were equivalent in terms of imagability and concreteness. Voluntary cue phrases were presented in the same format as no target cue phrases in the involuntary condition (i.e., centrally among horizontal line arrays).

All participants were presented with the same practice and experimental cue phrases in an identical sequential order, for involuntary and voluntary conditions, independently to which group (past, future) they were assigned. Cue phrases were originally developed by Schlagman and Kvavilashvili (2008) to reflect actual cues produced by participants in their diary studies and were translated from English to Danish using a professional translation service, then checked by the research team. In order to tailor the phrases to the participants' culture, British-specific phrases were changed to an appropriate Danish-specific alternative (e.g., from Telecom Tower to 


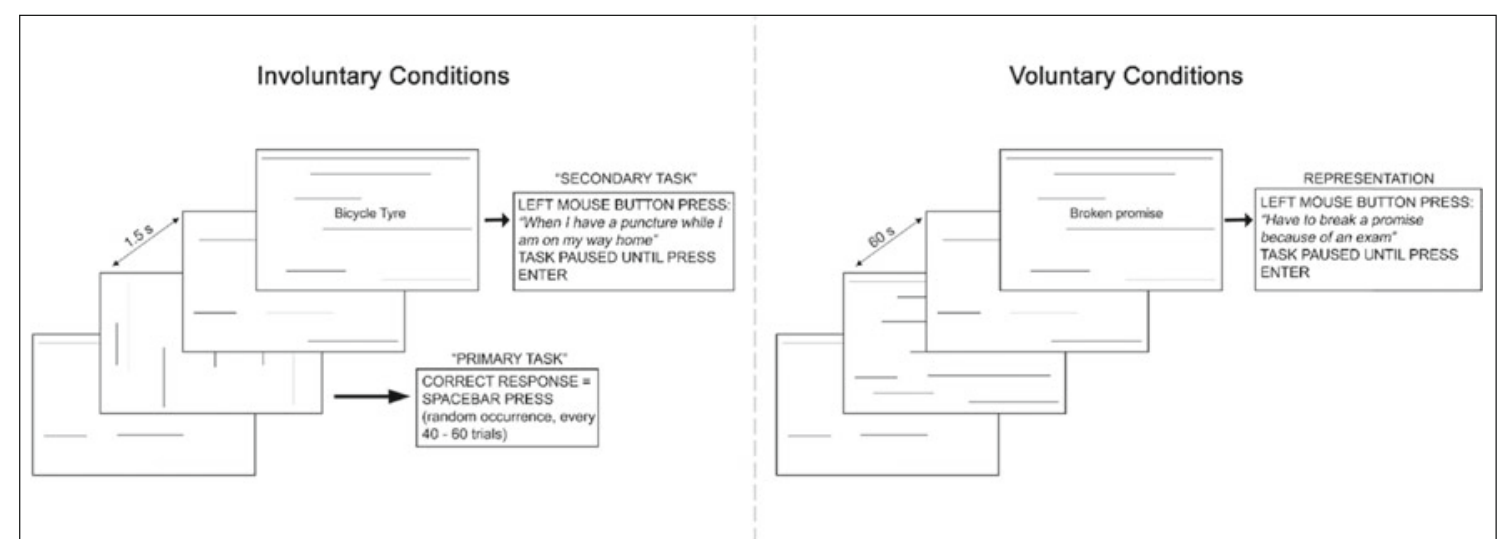

Fig. 1 Schematic Diagram of Experimental Procedure for Involuntary and Voluntary Conditions in the adapted Schlagman and Kvavilashvili (2008) paradigm

Rundetårn [a tower in Copenhagen]; from Mount Snowdon to Himmelbjerget [a well-known Danish hill]).

Shortened versions of cue phrase lists were used because, unlike Schlagman and Kvavilashvili (2008), both involuntary and voluntary conditions were presented in a single $1 \mathrm{hr}$ 45 min testing session, and therefore the overall number of trials needed to be shortened while avoiding floor effects. Pilot testing indicated that reducing the involuntary (800 to 600) and voluntary ( 24 to 12 ) cue phrases sufficiently reduced the session duration whilst eliciting a reliable number of past and future representations.

Autobiographical characteristics questionnaire A possible 24 representations could be entered in a single booklet (A4 size) with which each participant was provided, comprising involuntary representations on the first 12 pages and voluntary representations on the last 12 . Each event questionnaire was presented on a single page of the booklet (horizontally), consisting of two parts. Part 1 (on the left) was completed immediately after each event was elicited within the cue presentation phase. Participants were provided with space to write a short description of the event and a question concerning its vividness. In the involuntary conditions, participants were additionally asked to describe the event's trigger or cue, if known, and their level of concentration when the representation came to mind (see Appendix A). Part 2 was presented on the right of each sheet but was obscured with adhesive paper when Part 1 was completed. Part 2 required a more detailed written description and included a further 10 questions concerning specificity, temporal distance, emotion, mood impact, and autobiographical significance. To provide information on the extent that involuntary and voluntary future representations draw upon previous experience, we included two items concerning the novelty of future events: The extent to which the event or location had been experienced previously. These measures were included to verify that future events were not memories recast in a future orientation (for a related discussion, see Addis, Musicaro, Pan, \&
Schacter, 2010). Questions presented in Part 2 were identical for involuntary/voluntary conditions and were phrased according to their temporal orientation.

\section{Procedure}

Participants were tested individually with or without other participants in the same laboratory and completed experimental tasks in individual workstations. Each workstation comprised a desktop computer and a questionnaire booklet. After providing informed consent, an experimenter gave general administration instructions verbally to all participants. All other instructions were presented visually on E-Prime. Participants were informed that they could ask the experimenter questions once the program had started by silently raising their hands. Pilot testing ensured that slides provided clear and timely instructions; hence, participants very rarely asked questions.

First, on-screen instructions stated that participants would be required to perform a vigilance task, in which they were presented with slides with vertical and horizontal lines and should press the spacebar each time they identified a target (vertical lines). They were told to ignore nontargets (horizontal lines). Participants were also informed that, in addition to lines, they would see phrases, but they were to ignore these as these were to be detected by participants in another experimental condition (in actuality, no such condition existed). A 1min practice session (40 trials, three targets) was then completed.

Following the practice vigilance task, screen instructions differed depending on group assignment. The following refers to instructions provided in the future condition: Participants were first informed that since the task was monotonous they might think about other things, which was normal. Examples of such thoughts were provided, including goals, daydreams, and memories (the last example was "future representations" in the past condition). It was emphasized that they might 
experience future representations that "pop" into their mind spontaneously. In order to replicate the instructions from Schlagman and Kvavilashvili (2008) and to ensure a varied record of future-oriented thoughts, participants were told that future MTT could be near or far, specific or general (see Appendix B for the full description). Participants were told that, in addition to concentration task demands, they had to press the left mouse button when they had a future thought. They were told that this would pause the concentration task and to press enter to return to it. Each time the task was paused, text was presented informing participants that they should complete Part 1 of the questionnaire in the booklet and then press enter to return to the concentration task. The benefit of such a brief initial questionnaire was that individuals could give an immediate record of each retrieved event and its antecedent while providing an adequate event cue when completing Part 2. It also did not unnecessarily disrupt the primary ongoing concentration task. The concentration task took $15 \mathrm{~min}$ to complete but inevitably lasted longer based on the amount and length of pauses. If participants completed all 12 involuntary event questionnaires before the last slide, they continued the concentration task until it was completed (participants were never explicitly informed about the maximum number of events -12 per condition - to prevent expectation effects). All participants wore headphones in the involuntary phase for receiving audio feedback (a bell sound) with each spacebar press. The voluntary condition followed, which differed from the involuntary task in the following ways: Instructions highlighting involuntary elicitation were removed (see Appendix B), there was no parallel task, and participants consciously attempted to construct an event. If no left-mouse-button response was made after $1 \mathrm{~min}$, the next slide was automatically presented. A concluding slide informed participants they could have a refreshment break.

After approximately $5 \mathrm{~min}$ in an adjoining room, participants were handed a second booklet. This comprised three pages; a task list, Current Concerns Questionnaire, and Consideration of Future Consequences Questionnaire. Upon reentering the original room, each participant revisited their workstation and completed the Current Concerns Questionnaire, Part 2 of the Autobiographical Characteristics Questionnaire, and the Future Consequences scale, in that order. After removing the adhesive paper, participants completed all items of Part 2 (see Appendix A) for each representation recorded. Details of the Current Concerns and Future Consequences Questionnaires will not be described further as they are reported separately (Cole \& Berntsen, 2015). Cole and Berntsen addressed a separate research question concerning the relation between personal goals and MTT. The unique contribution of the present study concerns the application of an adapted laboratory paradigm to investigate involuntary MTT.

\section{Data analysis}

In order to calculate the latency for eliciting involuntary representations, the first author and a research assistant classified each trigger described by the participant (see Item 2 of Questionnaire, Appendix A) as cued by a word phrase, cued by other (e.g., current perceptions or feelings) or no cue (no cue identified). In circumstances when participants stated "cued by word" without specifying which cue phrase triggered the event, both coders checked for matches between cue phrases preceding the response. In all cases except five (which were not included in analyses, as neither rater could identify a related cue phrase), both raters agreed on which cue phrase matched each description. For example, the future event description "That I fail an exam" was matched with the cue phrase "fail an exam," and the past event description "stood and brushed my teeth, while I thought of me having kept my summer color pretty well" was matched to the cue phrase "healthy tan." Latency was only computed for representations that were triggered by one of the cue phrases. For the voluntary conditions, latency was simply the duration between cue phrase emergence and the participants' button press indicating a past/future representation. For continuous variables, we calculated means for each participant. For specificity, the proportion of representations classed as specific was calculated. For temporal distance, the proportion of a participant's representations was calculated within predefined temporal "bins" (see section below for details). For mood change, we had three categories (positive, neutral, and negative) and calculated proportions in each category per participants. Differences and interactions were analyzed using mixed factorial ANOVAs.

\section{Results}

\section{Descriptive data}

Participants completed the concentration task with reliably high levels of accuracy, identifying on average 10 out of 11 targets, which was invariant to group assignment (past, $M=$ $10.18, S D=0.95$; future, $M=9.85, S D=1.63$, Independent samples $t$ test, $t=-.91, p>.3, d=.25$ ). The mean level of concentration when involuntary representations were elicited was $3.07(S D=.92)$ for past MTT and $3.18(S D=.73)$ for future MTT ( 5 = full concentration), indicating that involuntary MTT arose in states of moderate concentration. Each representation was checked by the first author based on the criteria that each representation had to have a written valid description indicating an appropriate past or future representation and phenomenological ratings completed in Part 1 and 2 of the questionnaire. Ten past and three future representations did not fulfil these criteria, and were excluded. Of 
excluded past involuntary representations, two had insufficient ratings completed and six were not past-oriented (5) or personal (1). Of excluded voluntary past representations, two were not past oriented. Of the excluded future representations, one involuntary representation had insufficient ratings, one voluntary representation was a repetition, and the other was not future oriented. After participant and representation exclusions, there were 239 involuntary and 307 voluntary past representations and 154 involuntary and 269 voluntary future representations. Individuals produced $8.54(S D=3.85$, range $=1-12)$ involuntary and $10.96(S D=1.60$, range $=6-12)$ voluntary past representations, on average. The corresponding numbers for future representations was $5.70(S D=4.23$, range $=1-12)$ and $9.96(S D=1.79$, range $=6-12)$. The means and range scores show that future representations were less frequent than past representations, which was statistically significant, $F(1,53)=10.98, p<.005, \eta_{\mathrm{p}}{ }^{2}=.17$. Additionally, voluntary representations were significantly more numerous than those elicited involuntarily, $F(1,53)=30.68, p<.001$, $\left.\eta_{\mathrm{p}}{ }^{2}=.37\right)$ in line with findings from Schlagman and Kvavilashvili (2008). There was no interaction $\left(p=.14, \eta_{\mathrm{p}}{ }^{2}\right.$ $=.04$ ).

In terms of novelty $(1=$ maximum novelty, $5=$ minimum novelty), representations of the future were judged as moderately novel as they were only somewhat frequently similar to previous events (involuntary, $M=2.49, S D=1.01$; voluntary, $M=2.49, S D=0.60$ ). Also, future events somewhat frequently incorporated previously experienced settings (involuntary, $M=2.94, S D=1.09$; voluntary, $M=2.73, S D=0.88$ ). However, these data also showed that some involuntary and voluntary future events were not at all similar to previous experience (i.e., novel $[=1]$; involuntary $=34 \%$, voluntary $=34 \%$ ) and incorporated novel settings (involuntary $=27 \%$, voluntary $\left.=39 \%, \chi^{2}=4.92, p<.05\right)$. This descriptive data suggests that whereas both involuntary and voluntary future thoughts contain a similar amount of novel events, the novelty of the event location may be dependent on retrieval mode: Specifically, voluntary future thoughts (versus involuntary future thoughts) tend to involve more novel locations.

\section{Cues}

Examination of the cues demonstrated that of all involuntary past representations, $69 \%$ were triggered by the word phrases, $8 \%$ by other aspects of thoughts or the environment, and $23 \%$ had no known trigger, showing that, similar to Schlagman and Kvavilashvili (2008), the vast majority of identified triggers were cue phrases from the concentration task. Importantly, the respective percentages for the involuntary future representations were $58 \%, 12 \%$, and $29 \%$, showing a similar high percentage of stimuli-triggered representations (cf. Berntsen \& Jacobsen, 2008).

\section{Effects of involuntary versus voluntary retrieval}

Here we examine our predictions that involuntary representations will be elicited faster and will more likely be specific and emotionally impactful, regardless of whether the representation is oriented in the past or future. As latency data was positively skewed, log transformations were carried out on all data, which normalized the reaction time distributions following recommendations by Ratcliff (1993). The ANOVA analysis of latency (using the transformed data, all ShapiroWilk statistics, $p>.05$ ) demonstrated that, on average, involuntary representations were retrieved faster than voluntary representations, and there was a large significant difference $\left(\eta_{\mathrm{p}}{ }^{2}=.71\right.$; see Table 1 for ANOVA statistics). Examination of the untransformed means and standard deviations in Table 1 confirms a difference of, on average, approximately eight seconds resulting from the retrieval mode manipulation (3.39 s vs. $11.27 \mathrm{~s})$.

Phenomenological variables indicated several reliable differences between involuntary and voluntary MTT. First, confirming our hypothesis and previous findings, involuntary MTT was more frequently categorized as specific than voluntary MTT (see Table 1): Whereas $70 \%$ of involuntary MTT was rated as specific, this was only true for $52 \%$ of voluntary MTT $\left(\eta_{\mathrm{p}}{ }^{2}=.27\right)$. Second, involuntary MTT was rated lower on vividness than voluntary MTT $\left(\eta_{\mathrm{p}}{ }^{2}=.25\right.$; see Table 1$)$. Third, involuntary MTT was significantly more rehearsed than voluntary MTT $\left(\eta_{\mathrm{p}}{ }^{2}=.38\right.$; see section below and Table 1 for interaction effect). Fourth, in line with predictions, involuntary MTT was more likely to elicit mood impact than voluntary MTT $\left(\eta_{\mathrm{p}}{ }^{2}=.08\right.$; see No Mood Impact variable, Table 1$)$. Additionally, there were significant interactions between temporal direction and retrieval mode concerning positive and negative mood impact (see Table 1). Fifth, involuntary MTT was judged as more emotionally intense than voluntary MTT $\left(\eta_{\mathrm{p}}{ }^{2}=.23\right)$. In terms of personal significance, it was found that involuntary MTT was considered less central to life story than voluntary MTT $\left(\eta_{\mathrm{p}}{ }^{2}=.07\right.$; see Table 1$)$. Last, involuntary MTT was dated closer to the present than voluntary MTT $\left(\eta_{\mathrm{p}}^{2}=.09\right.$; see Table 1$)$.

In terms of rehearsal frequency, a significant interaction emerged from the higher rehearsal ratings for involuntary future MTT over and above all other event categories, which was demonstrated formally by follow-up comparisons (all $p \mathrm{~s}$ $<.001$; see Table 1 for means, all follow-up comparisons used Bonferroni corrections).

In terms of valence ratings, there was an interaction that can be deconstructed as follows: Voluntary past and future MTT valence ratings were largely equivalent, but the effect of the involuntary mode on past and future MTT caused them to differ in opposite directions on valence (past $=$ less positive, future $=$ more positive; see Table 1). Although all averages were on the positive side of the scale, the range of valence 
Table 1 Characteristics of Past and Future MTT as a Function of Retrieval Mode ${ }^{+}$Statistical trend $(p<.08)$

\begin{tabular}{|c|c|c|c|c|c|c|c|}
\hline & \multicolumn{2}{|l|}{ Past } & \multicolumn{2}{|l|}{ Future } & \multicolumn{3}{|c|}{$F$ values from mixed ANOVAs } \\
\hline & Involuntary & Voluntary & Involuntary & Voluntary & Past vs. Future & Involuntary vs. Voluntary & Interaction \\
\hline Reaction Time $(\mathrm{ms})^{\times}$ & $3,149(2002)$ & $9,665(5778)$ & $3,623(2215)$ & $13,870(8315)$ & 2.79 & $103.68 * * *$ & 0.14 \\
\hline Specificity $(0-1)$ & $.70(.27)$ & $.58(.19)$ & $.69(.31)$ & $.46(.29)$ & 1.06 & $19.25^{* * *}$ & 1.95 \\
\hline Vividness (1-7) & $3.87(1.07)$ & $4.60(0.95)$ & $3.48(1.26)$ & $4.03(1.03)$ & $3.74^{+}$ & $17.98 * * *$ & 0.34 \\
\hline Temporal Distance (years) & $4.42(5.02)$ & $5.05(2.95)$ & $1.63(2.31)$ & $4.35(6.38)$ & $3.37^{+}$ & $5.10^{*}$ & 1.99 \\
\hline Rehearsal (1-5) & $2.74(0.74)$ & $2.41(0.57)$ & $3.58(0.89)$ & $2.58(0.73)$ & $9.81 * * *$ & $31.93 * * *$ & $7.94 * *$ \\
\hline Positive Mood Impact (0-1) & $.38(.26)$ & $.42(.20)$ & $.55(.33)$ & $.42(.19)$ & 2.51 & 0.98 & $4.17^{*}$ \\
\hline Negative Mood Impact (0-1) & $.24(.23)$ & $.13(.14)$ & $.15(.15)$ & $.17(.14)$ & 0.37 & 1.84 & $4.21 *$ \\
\hline No Mood Impact (0.0-1.0) & $.38(.28)$ & $.45(.21)$ & $.30(.33)$ & $.41(.25)$ & 0.96 & $4.69 *$ & 0.20 \\
\hline Valence $(-2-+2)$ & $0.16(0.80)$ & $0.43(0.41)$ & $0.79(0.66)$ & $0.54(0.37)$ & $11.62 * * *$ & 0.02 & $5.16^{*}$ \\
\hline Emotional Intensity (1-5) & $2.78(0.71)$ & $2.19(0.63)$ & $2.85(0.91)$ & $2.57(0.68)$ & 1.92 & $15.63 * * *$ & 2.06 \\
\hline Central to Life Story (1-5) & $2.23(0.76)$ & $2.08(0.64)$ & $2.54(1.13)$ & $2.13(0.72)$ & 1.06 & $4.02 *$ & 0.85 \\
\hline Importance to Identity $(1-5)$ & $2.12(0.84)$ & $1.99(0.75)$ & $2.44(1.16)$ & $2.06(0.64)$ & 1.15 & $3.35^{+}$ & 0.80 \\
\hline
\end{tabular}

${ }^{\times}$For this analysis only, the number of participants included was reduced (past, $N=25$, future, $N=19$ )

${ }^{*} p<.05 . * * p<.01 . * * * p<.005$

ratings indicated that involuntary representations were less homogenous across temporal directions, containing more highly positive/highly negative representations than voluntary representations (ranges: involuntary past; $-2.0-+1.8$ involuntary future, $-1.0-+2.0$; voluntary past: $-0.5-1.1$; voluntary future: $0.0-1.7$; Scale anchor points, $-2-+2$ ).

\section{Effects of temporal direction (past versus future)}

Prior research concerning effects of temporal direction converged on five hypotheses: That past representations would be more vivid and more specific but less positive, less important to the self, and temporally further away than future representations. Latency analysis indicated no main effect of temporal direction (see ANOVA results on Table 1). ${ }^{1}$ Turning to phenomenological variables, the difference between past and future MTT on vividness indicated that past MTT was more vivid than future MTT, in line with predictions, but, like Berntsen and Jacobsen (2008), did not reach the criteria for statistical significance $\left(p=.059, \eta_{\mathrm{p}}{ }^{2}=.07\right.$; see Table 1$)$. Second, a significant future positivity bias was found when compared with the past $\left(\eta_{\mathrm{p}}{ }^{2}=.18\right.$; see Table 1$)$. Third, future MTT was significantly more rehearsed than past MTT $\left(\eta_{\mathrm{p}}{ }^{2}=.16\right.$; see Table 1). There was no temporal direction effect upon specificity.

As shown in Table 1, no main effects of temporal direction were found for temporal distance. However, this analysis -

\footnotetext{
${ }^{1}$ However, within an independent samples $t$ test, the difference between voluntary past and future approached significance, $t(56)=1.89, p=.064$, $d=.52$.
}

based on means - did not address whether the distributions might vary. We therefore assessed temporal distance within a temporal distribution of responses, using representations as the unit of analysis: The dependent variable here is proportion of representations elicited of the total amount of dated representations in that particular condition (e.g., involuntary past), as each condition contained unequal numbers of representations. ${ }^{2}$ We analyzed the temporal distribution, as it illustrates whether the distribution of past and future representations mirror each other and the degree of variation in the distribution of representations across the four representation types, if any (cf. Berntsen \& Jacobsen, 2008; Spreng \& Levine, 2006). Figure 2 illustrates the temporal distance of representations in each condition, subdivided into frequencies of representations in six temporal bins $(0,1,2,3,4,>5$ years from present). As Fig. 2 illustrates, a greater proportion of future representations were close to the present than past representations (64\% vs. $32 \%$, where 1 year from present represents temporally close) and future representations elicited in an involuntary mode were somewhat more likely to be within 1 year from present than voluntary ones ( $68 \%$ vs. $60 \%)$. For past representations, the proportions for involuntary and voluntary representations were more similar (33\% vs. $31 \%$, respectively). To formally assess these differences, a mixed ANOVA using proportion of representations within 1 year from present was conducted, restricting analysis to temporally close representations. This confirmed that future representations were significantly more likely to be temporally close than past representations, $F(1$,

\footnotetext{
${ }^{2}$ This is in contrast to Berntsen and Jacobsen (2008), who used absolute frequencies due to having equivalent numbers of responses in each condition.
} 


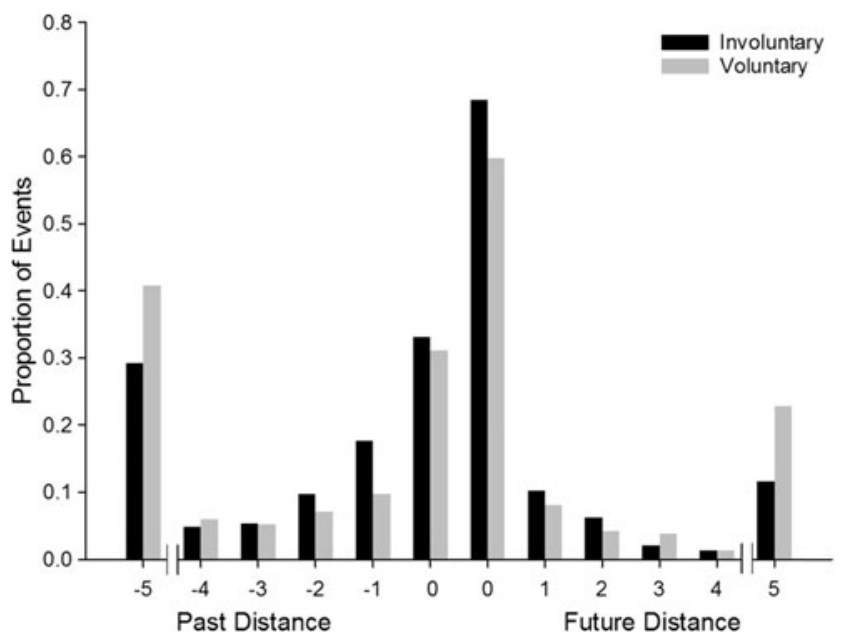

Fig. 2 Temporal distribution of representations across four conditions

$53)=17.02, p<.001, \eta_{\mathrm{p}}^{2}=.24$. Although there was a greater difference in proportions for future than past representations in the involuntary condition, no interaction was found, $F(1,53)=$ $0.74, p=.39, \eta_{\mathrm{p}}^{2}=.01$. Neither was there a main effect of retrieval mode, $F(1,53)=0.57, p=.45, \eta_{\mathrm{p}}{ }^{2}=.01$. From these analyses, we concluded that there are a greater proportion of future representations close to the present but that the involuntary mode does not further increase this proportion in comparison with past representations. Thus, involuntary future representations are numerically but not statistically closer to the present than voluntary future representations (see Berntsen \& Jacobsen, 2008, for a similar finding).

\section{The relation between event valence and mood change}

To assess whether emotional valence of the event representation was associated with congruent changes in mood, four Pearson product-moment correlations were carried out at the representation level (using a $-1,0$, and +1 three-point scale of mood impact). As predicted, these correlations showed a tight correspondence between event valence and mood impact: Across the four event types, event valence was significantly and positively correlated with mood change (range of $r \mathrm{~s}$ $=.76-.87$; all $p$ s $<.001$ ).

However, these item analyses do not take into consideration interindividual differences, which may impact on the relation between event valence and mood change. In a further multiple regression analysis examining whether emotional valence was a significant predictor of mood change, we were able to control for the interindividual variance by dummy coding for $N-1$ participants in each condition (Cohen \& Cohen, 1983, e.g., see also, Berntsen \& Bohn, 2010, for a similar analytical approach). Mood change was entered as the dependent variable in each of the four multiple regression models, with event valence as a predictor (together with 26/27 dummy-coded variables to control for subject variance). This analysis showed that for all conditions, valence was a significant positive predictor of mood change - involuntary future, $\beta=.92, t(26)=21.04, p<.001 ;$ voluntary future, $\beta=.81$, $t(26)=21.43, p<.001$; involuntary past, $\beta=.79, t(27)=$ $18.00 p<.001$, voluntary past, $\beta=.74, t(27)=19.39, p<$ .001 ; standardized $\beta$ s reported.

\section{Discussion}

In this study, we introduced a new laboratory paradigm for examining voluntary and involuntary future and past MTT. This was achieved by adapting a paradigm originally designed to study autobiographical memory (Schlagman \& Kvavilashvili, 2008). This paradigm was validated by replicating and extending the findings of prior studies utilizing naturalistic methods showing differences between involuntary versus voluntary MTT (Berntsen \& Jacobsen, 2008; Finnbogadóttir \& Berntsen, 2011). Additionally, we replicated differences between past versus future voluntary representation and extended these to involuntary representations elicited in the laboratory.

As with involuntary memories, it was found that involuntary future representations had identifiable cues and occurred when participants were in a state of moderate concentration (see also Berntsen \& Jacobsen, 2008; Finnbogadóttir \& Berntsen, 2011). The fact that this was demonstrated within a controlled setting rather than in the context of everyday life (Berntsen \& Jacobsen, 2008; Finnbogadóttir \& Berntsen, 2011), underscores the robustness of this result and illustrates its generality. We also replicated the finding that involuntary representations more regularly concerned specific events (e.g., Berntsen, 1998; Berntsen \& Hall, 2004; Berntsen \& Jacobsen, 2008; Mace, 2006) and elicited a greater emotional impact (see Berntsen \& Jacobsen, 2008; Berntsen, 2009, for a review). We reported a new finding regarding the content of involuntary future thoughts, indicating that they involve a similar amount of novel events (i.e., events that had never happened in the past) as the ones constructed voluntarily, suggesting they are not simple "replays" of past experiences.

Importantly, the paradigm enabled us to elucidate involuntary future MTT in finer detail and investigate previously unmeasured dimensions. The current study shows that involuntary future projections arise in consciousness rapidly (approximately $3 \frac{1}{2}$ seconds); which is comparable with involuntary memories, and in sharp contrast with voluntary past and future representations that took on average around 10 to 14 seconds to generate.

We also replicated and extended key findings from MTT research. Specifically, we found a future positivity bias, whereby future representations, in general, were rated as more positive than their mnemonic counterparts. This is consistent amongst studies of voluntary (e.g., Berntsen \& Bohn, 2010) 
and involuntary (Bernstein \& Jacobsen, 2008) MTT. However, this effect may only arise when participants freely select past and future events: A recent study found a reversal of this bias when only negative events are requested (Rubin, 2014), suggesting that both effects may reflect schema-based constructions. Another robust finding is that past events are more subjectively vivid than future ones (Berntsen \& Bohn, 2010; D'Argembeau \& Van der Linden, 2004, 2006; Gamboz et al., 2010; Hassabis et al., 2007). However, the present study found only a trend in the predicted direction. Finally, similar to previous findings inside (Addis et al., 2008) and outside (Berntsen \& Jacobsen, 2008; Finnbogadóttir \& Berntsen, 2011; Miles \& Berntsen, 2011) the laboratory, we found that mental representations of the future were temporally closer to the present than representations of the past. However, we did not replicate the finding that future MTT was more important to the self, on either the identity or life story measures (Addis et al., 2008; Berntsen \& Bohn, 2010; although, see Berntsen \& Jacobsen, 2008) and we did not find that past MTT was more specific than future MTT (Anderson \& Dewhurst, 2009; Berntsen \& Jacobsen, 2008; Miles \& Berntsen, 2011).

The result concerning the difference in response latency between voluntary past and future thinking, showing a nonsignificant — but still moderate (Cohen, 1988) — effect in the predicted direction, arose in the context of previous inconclusive results (e.g., Anderson et al., 2012; Addis et al., 2009). Contributing to these findings are neuroimaging studies indicating more cognitive effort for future versus past events (see Schacter et al., 2012, for a review). It is possible that the cognitive effort associated with future thinking depends upon moderating factors, such as the degree of schema-based construction (Berntsen \& Bohn, 2010), event novelty, or the extent of novel recombination (see Addis et al., 2010). In fact, the present findings suggest that strategically constructed future events may allow the construction of events with unfamiliar locations, whereas this is more unlikely under involuntary conditions. Also, a study of older adults suggests that it may be more demanding to imagine events drawn from disparate episodic details (Addis et al., 2010). This suggests future studies should measure factors potentially moderating cognitive effort.

Clearly evident, however, was the effect of retrieval mode upon reaction time, which is consistent with previous findings as well as neuroimaging data demonstrating increased activity in prefrontal regions using a voluntary, compared with involuntary, retrieval mode (Hall et al., 2014). The present study presents behavioural data showing that the retrieval dynamics of involuntary future MTT are highly similar to those of involuntary memories. This agrees with the aforementioned idea that involuntary MTT relies on an interaction between cues in the environment/thought and autobiographical knowledge (Berntsen, 2009), which occurs more rapidly than in construction of voluntary MTT, the latter mediated by strategic control processes.

We also found some novel, unpredicted results, which may be important for understanding the nature of involuntary MTT. Key among these concern emotion. The present study suggests that, in healthy adults, an asymmetry exists in the emotional aspects of past and future involuntary MTT that is not present in voluntary MTT: Involuntary memories were less positive, associated with a more negative mood impact than future counterparts, which were, in turn, more positive, associated with a more positive mood impact. However, this finding should be interpreted with caution since it has not been reported in previous research (Berntsen \& Jacobsen, 2008; Finnbogadóttir \& Berntsen, 2011) which found only main effects of retrieval mode on emotional valence and mood impact. Considering this is the first laboratory study of its kind, it will be important to conduct further diary and laboratory investigations, perhaps in a single sample, to account for these discrepancies. However, it is noteworthy that, consistent with previous work (Berntsen, 1996), event valence significantly predicted mood change across all conditions. The fact that involuntary past and future thinking has the potential to impact mood could have implications for understanding emotion regulation in healthy and clinical groups (e.g., depressed individuals). This would therefore be an area worthy of investigation.

The result indicating that the future was more rehearsed than the past, exaggerated in the involuntary mode, was also not predicted and is inconsistent with some previous studies of MTT that found increased rehearsal frequency for past events (Berntsen \& Jacobsen, 2008; Finnbogadóttir \& Berntsen, 2011; Miles \& Berntsen, 2011). The present finding could be explained in two ways. As future thoughts rely more heavily on schema (Berntsen \& Bohn, 2010; Rubin, 2014), they may contain regularities which are more rehearsed. On the other hand, due to being more goal salient (see Cole \& Berntsen, 2015; Schacter, 2012), involuntary future projections could more likely be based on uncompleted goals than involuntary memories. Under this view, rehearsal is important in goal maintenance and completion.

The study of involuntary future representations prompts an important theoretical question: From which sources do involuntary future representations originate? One account first expressed in Berntsen and Jacobsen (2008) is that involuntary future representations are elicited in a bottom-up fashion by cues and thereafter enact a fast spreading activation across associative nodes within one's autobiographical memory, similar to when a personal memory is recalled (see Conway \& Pleydell-Pearce, 2000). This representation is then judged as future oriented rather than originating from the personal past using semantic knowledge. An alternate explanation is that involuntary future representations were originally voluntary constructions, and are therefore memories of future thoughts. 
The latter explanation appears contradicted by the fact that only a very small percentage of involuntary future projections were rated as being frequently experienced in the past $(12 \%$; see also Addis et al., 2009). Also, this, along with the fact that the definition of future thought was fully explained before the vigilance task, works against the idea that involuntary future representations are actually involuntary memories recast in a future direction (which might happen for events that could happen regularly in the past or future); a common criticism of voluntarily constructed future thoughts (see Addis et al., 2010). We believe it is premature to arrive at a firm conclusion on this important theoretical question. Therefore, we highlight these alternatives (which may not be mutually exclusive), so that empirical studies begin to test them.

We also note that, due to its emphasis on off-task thought processes, it may be tempting to draw comparisons between the results described here and those found in the literature on mind wandering (see Baird, Smallwood, \& Schooler, 2011). However, there are important conceptual differences between these phenomena: Involuntary MTT into the past and future (but not mind wandering per se) is noticeably autobiographical, typically cue dependent, short-lived, and not necessarily off-task thinking. For example, involuntary autobiographical memories can be triggered by, and serve important functions for, an ongoing task, such as reading or problem solving, as evidenced by the literature on spontaneous episodic remindings (Hintzman, 2011; Miles \& Berntsen 2015; Schank, 1982), whereas mind wandering is defined as offtask thinking. Also, involuntary MTT is unintentional by definition, whereas mind wandering can be volitional in that the person can intentionally choose to disengage from an external task in order to pursue an internal stream of thought (e.g., McMillan, Kaufman, \& Singer, 2013). Furthermore, sustained mind wandering involves the recruitment of a frontal-parietal control network (Smallwood, Brown, Baird, \& Schooler, 2012), whereas involuntary remembering take place with little brain activation associated with strategic control (Hall et al., 2014). In our view, these distinguishing features preclude direct comparison between these two phenomena. See Berntsen, Rubin and Salgado (2015) for a more elaborated discussion.

\section{Limitations}

Over and above the benefits already assigned to this paradigm, the analysis of frequency across conditions shows that this paradigm can be utilized to reliably elicit enough involuntary future representations for analysis of latency and subjective characteristics. Still, some limitations should be acknowledged. First, as a primary aim of this study was to extend the paradigm by Schlagman and Kvavilashvili (2008), we necessarily maintained a within-group factor of retrieval mode rather than temporal direction. Yet, one could argue that carryover effects from involuntary to voluntary conditions confounded our results and that a between-groups design was required. However, systematic differences between the involuntary and voluntary conditions were found that render carryover effects less likely, since these naturally would have worked against the observation of differences between the conditions. Second, one could argue that circumstantial differences between involuntary and voluntary retrieval mode conditions affected the observed effects rather than their inherent cognitive process. In relation to this point, one might note differences in amount of cues between involuntary and voluntary conditions (600 vs. 12). However, the present findings were consistent with diary studies of involuntary and voluntary MTT in which participants were asked to retrieve a fixed number of involuntary and voluntary memories/future events for each of the four conditions (Berntsen \& Jacobsen, 2008; Finnbogadóttir \& Berntsen, 2011). More generally, the present results also agree with a substantial body of involuntary memory research (see Berntsen, 2009, for a review). Also relating to the above point, a potential limitation to the methods applied is the difference between the involuntary and voluntary conditions, primarily, the lack of a parallel distraction task in the voluntary condition. However, in a recent comparison of voluntary and involuntary episodic memory, a parallel task in the voluntary condition was included to examine this possible confounder (Berntsen et al., 2013). The parallel task appeared to interfere with the voluntary search process, thus dramatically increasing retrieval time (and hence increasing this difference between involuntary and voluntary conditions) while keeping retrieval frequencies intact. This suggests that adding a parallel task to the voluntary conditions in the present study most likely would have intensified the observed differences between the involuntary and voluntary conditions rather than diminished them.

Finally, it could be argued that making participants aware of the existence of involuntary MTT (in the past and future groups) could increase their frequency or, importantly, alter their phenomenological characteristics (cf. Vannucci, Batool, Pelagatti, \& Mazzoni, 2014, for an examination of this issue in involuntary memories). The potential for reactivity in this aspect of the present design means that future experiments should attempt to replicate these initial findings using a condition in which no mention is made of involuntary MTT prior to the vigilance task. This should help determine whether the differences between temporal direction and retrieval mode found here hold across these varying procedural components. Future studies may also benefit from using self- and probecaught methods of assessing involuntary thoughts (Smallwood \& Schooler, 2006). Consistent findings across 
such design alterations should bolster the empirical findings described here.

\section{Future directions}

This study opens new questions concerning the phenomena of involuntary future MTT. First, why do some cues trigger future representations whereas others do not? One possibility is that cues are more likely to elicit future MTT when they interact with current goal states or that a certain level of cue discriminability is needed (Berntsen et al., 2013). However, further experimentation is required to examine these possibilities (see Schlagman \& Kvavilashvili, 2008, for arguments concerning how cues and memory interact in involuntary past MTT; see Berntsen et al., 2013, for manipulations of cueing in relation to activating involuntary episodic memories). Another, but related, question concerns the role of unconscious processes in involuntary future thinking; specifically, the extent to which they are primed by previous thoughts or goals (see Szpunar, 2010). The present paradigm might also be developed to examine the emotional characteristics of involuntary MTT experiences in psychological disorders, such as depression and PTSD (see Berntsen \& Rubin, 2014).

\section{Summary}

We adapted a laboratory paradigm (Schlagman \& Kvavilashvili, 2008) to examine past and future MTT in both involuntary and voluntary modes. Contrasting with diary studies, the paradigm ensured that many aspects of the context were held constant (e.g., location, presentation of cues) while only the key variables of interest were manipulated. We successfully replicated and extended data on involuntary MTT reported in naturalistic studies by analyzing subjective characteristics and response times. This study represents the first step to experimentally investigate involuntary future MTT, leading to numerous tractable avenues for future research.

Author Note The authors wish to thank the Danish National Research Foundation (DNRF93) for funding. We are thankful to Lia Kvavilashvili for providing extensive experimental materials. We would also like to thank Daniella Villadsen, Thorbjørn Larsen, and Marie Kirk for assisting in participant recruitment and conducting the study. In addition, we would like to thank Dorthe Thomson and Jonathan Koppel for their constructive comments to an earlier version of this manuscript, and Sinue Salgado for help with Figures. The authors affirm no conflict of interest.

\section{Appendix A}

Items on the Autobiographical Questionnaire for Involuntary and Voluntary Imagined Future Events (except where stated).

Part One (immediate responses)
1.[Vividness] Indicate how lifelike the imagined future event was (1-7, 1 = vague almost no image, $7=$ very vivid almost like normal vision)

2.[Cue] Was the imagined future event triggered by anything (in your thoughts or in the environment)? If so please describe the trigger*

3. [Concentration] Please rate on the scale below how much you were concentrating on the concentration task when the imagined future event came to mind $(1-5,1=$ not at all concentrating, $5=$ fully concentrating)*

Part Two (retrospective responses)

1.[Specificity] Does this representation refer to a particular situation in a particular day in your future? (yes/no)

2a.[Temporal Distance: Age-at-event] How old are you in the imagined future event? (age estimated in years)

2b.[Temporal Distance in days] If less than a year, how many days from present? (distance estimated in days)

3.[Event Novelty] How often have you previously experienced the same or similar event?

$(1-5,1=\text { never, } 5=\text { very often })^{\#}$

4.[Location Novelty] How often have you personally experienced the same setting of the event before?

$(1-5,1=\text { never, } 5 \text { = very often })^{\#}$

5.[Rehearsal] How often have you previously thought about the imagined future event

$(1-5,1=$ never, $5=$ very often $)$

6.[Emotional Valence] Is the imagined future event positive or negative?

$(-2+2,-2=$ very negative,$+2=$ very positive $)$

7.[Emotional Intensity] Is the imagined future event an emotionally intense situation?

$(1-5,1=$ no intensity, $5=$ very intense $)$

8.[Mood Impact] Did the imagined future event affect your mood? (better, worse, neutral)

9.[Life story] I feel this event will become a central part of my life story $(1-5,1=$ totally disagree, $5=$ totally agree $)$

10.[Identity] I feel this event will become part of my personal identity $(1-5,1=$ totally disagree, $5=$ totally agree $)$

Note. For the past MTT condition, the wording was changed so that each question referred to the past. In Part two, Item 3 is adapted from a study by Gamboz, Brandimonte, and deVito (2010).

*Presented only for involuntary past/future MTT.

\# Not analyzed in any past MTT condition.

\section{Appendix B}

Voluntary future MTT was described to participants as follows:

"(Spontaneous) future thoughts can be in the very near future or the distant future. They can vary in relation to detail and specificity. A specific future thought refers to a single episode of your life (e.g., a visit with a family member in the hospital, or when a new baby is born). A general future thought can refer to an extended event (e.g., multiday trip to Paris) or a single event that might happen repeatedly over a longer period (e.g., repeated visits to the dentist, or go to the beach every summer when I start a family). It may be an idea 
about the future you've had many times before, or an idea you've never had before. (The most important thing is that your conception of the future came to you SPONTANEOUSLY, i.e., without you consciously trying to think of something)."

Note. Text in parentheses was added in the involuntary condition and hence were the only difference in the description of a future representation between involuntary and voluntary conditions. Instructions were identical for the past conditions except references to time, which were modified to indicate a memory.

\section{References}

Addis, D. R., Pan Musicaro, L., \& Schacter, D. L. (2010). Episodic simulation of past and future events in older adults: Evidence from an experimental recombination task. Psychology and Aging, 25, 369-376.

Addis, D. R., Pan, L., Vu, M.-A., Laiser, N., \& Schacter, D. L. (2009). Constructive episodic simulation of the future and the past: Distinct subsystems of a core brain network mediate imagining and remembering. Neuropsychologia, 47, 2222-2238. doi:10.1016/j. neuropsychologia.2008.10.026

Addis, D. R., Wong, A., \& Schacter, D. L. (2007). Remembering the past and imagining the future: Common and distinct neural substrates during event construction and elaboration. Neuropsychologia, 45, $1363-1377$

Addis, D. R., Wong, A., \& Schacter, D. L. (2008). Age-related changes in the episodic simulation of future events. Psychological Science, 19, 33-41.

Anderson, B. A., Jacoby, L. L., Thomas, R. C., \& Balota, D. A. (2011). The effects of age and divided attention on spontaneous recognition. Memory \& Cognition, 39, 725-735.

Anderson, R. J., \& Dewhurst, S. A. (2009). Remembering the past and imagining the future: Differences in event specificity of spontaneously generated thought. Memory, 17, 367-373. doi:10.1080/ 09658210902751669

Anderson, R. J., Dewhurst, S. A., \& Nash, R. (2012). Shared processes underlying past and future thinking: The impact of imagery and concurrent task demands on event specificity. Journal of Experimental Psychology: General, 38, 356-365. doi:10.1037/ a0025451

Baird, B., Smallwood, J., \& Schooler, J. W. (2011). Back to the future: Autobiographical planning and the functionality of mind-wandering. Consciousness \& Cognition, 20, 1604-1611. doi:10.1016/j. concog.2011.08.007

Ball, C. T. (2007). Can we elicit involuntary autobiographical memories in the laboratory? In J. H. Mace (Ed.), Involuntary memory (pp. 127-153). Oxford, England: Blackwell.

Berntsen, D. (1996). Involuntary autobiographical memories. Applied Cognitive Psychology, 10, 435-454.

Berntsen, D. (1998). Voluntary and involuntary access to autobiographical memory. Memory, 6(2), 113-141.

Berntsen, D. (2009). Involuntary autobiographical memories: An introduction to the unbidden past. Cambridge, England: Cambridge University Press.

Berntsen, D. (2010). The unbidden past: Involuntary autobiographical memories as a basic mode of remembering. Current Directions in Psychological Science, 19, 138-142. doi:10.1177/ 0963721410370301

Berntsen, D., \& Bohn, A. (2010). Remembering and forecasting: The relation between autobiographical memory and episodic future thinking. Memory \& Cognition, 38, 265-278. doi:10.3758/MC.38. 3.265

Berntsen, D., \& Hall, N. M. (2004). The episodic nature of involuntary autobiographical memories. Memory \& Cognition, 32, 789-803.

Berntsen, D., \& Jacobsen, A. S. (2008). Involuntary (spontaneous) mental time travel into the past and future. Consciousness \& Cognition, 17, 1093-1104. doi:10.1016/j.concog.2008.03.001

Berntsen, D., \& Rubin, D. (2014). Pre-traumatic stress reactions in soldiers deployed to Afghanistan. Clinical Psychological Science. doi: $10.1177 / 2167702614551766$

Berntsen, D., Staugaard, S. R., \& Sørensen, L. M. T. (2013). Why am I remembering this now? Predicting the occurrence of involuntary (spontaneous) episodic memories. Journal of Experimental Psychology: General, 142(2), 426-444. doi:10.1037/a0029128

Berntsen, D., Rubin, D. C., \& Salgado, S. (2015). The frequency of involuntary autobiographical memories and future thoughts in relation to daydreaming, emotional distress, and age. Cognition \& Consciousness, 36, 352-372.

Berryhill, M. E., Picasso, L., Arnold, R., Drowos, D., \& Olson, I. R. (2010). Similarities and differences between parietal and frontal patients in autobiographical and constructed experience tasks. Neuropsychologia, 48, 1385-1393. doi:10.1016/j. neuropsychologia.2010.01.004

Cohen, J. (1988). Statistical power analysis for the behavioral sciences (2nd ed.). Hillsdale, NJ: Erlbaum.

Cohen, J., \& Cohen, P. (1983). Applied multiple regression/correlation analysis for the behavioural sciences (2nd ed.). Hillsdale: NJ: Erlbaum.

Cole, S. N., \& Berntsen, D. (2015). Do future thoughts reflect personal goals? Current concerns and mental time travel into the past and future. Quarterly Journal of Experimental Psychology. doi:10. 1080/17470218.2015.1044542

Cole, S. N., Morrison, C. M., \& Conway, M. A. (2013). Episodic future thinking: Linking neuropsychological performance and episodic detail in young and old Adults. Quarterly Journal of Experimental Psychology, 66, 1687-1706. doi:10.1080/17470218.2012.758157

Conway, M. A. (2005). Memory and the self. Journal of Memory and Language, 53, 594-628. doi:10.1016/j.jml.2005.08.005

Conway, M. A., \& Pleydell-Pearce, C. W. (2000). The construction of autobiographical memories in the self-memory system. Psychological Review, 107, 261-288.

Crovitz, H. F., \& Schiffman, H. (1974). Frequency of episodic memories as a function of their age. Bulletin of the Psychonomic Society, 4(NB5), 517-518.

D’Argembeau, A., \& Van der Linden, M. (2004). Phenomenal characteristics associated with projecting oneself back into the past and forward into the future: Influence of valence and temporal distance. Consciousness \& Cognition, 13, 844-858. doi:10.1016/j.concog. 2004.07.007

D’Argembeau, A., \& Van der Linden, M. (2006). Individual differences in the phenomenology of mental time travel: The effect of vivid visual imagery and emotion regulation strategies. Consciousness \& Cognition, 15, 342-350. doi:10.1016/j.concog.2005.09.001

Ericsson, K. A., \& Simon, H. A. (1980). Verbal reports as data. Psychological Review, 87, 215-251.

Finnbogadóttir, H., \& Berntsen, D. (2011). Involuntary and voluntary mental time travel in high and low worriers. Memory, 19(6), 625640. doi:10.1080/09658211.2011.595722

Finnbogadóttir, H., \& Berntsen, D. (2013). Involuntary future projections are as frequent as involuntary memories, but more positive. Consciousness \& Cognition, 22, 272-280.

Gamboz, N., Brandimonte, M. A., \& de Vito, S. (2010). The role of past in the simulation of autobiographical future episodes. Experimental Psychology, 57, 419-428. doi:10.1027/1618-3169/a000052

Hall, S. A., Rubin, D., Miles, A., Davis, S. W., Wing, E. A., Cabeza, R., \& Berntsen, D. (2014). The neural basis of involuntary episodic 
memories. Journal of Cognitive Neuroscience, 26(10), 1-15. doi:10. 1162/jocn_a_00633

Hassabis, D., Kumaran, D., \& Maguire, E. A. (2007). Using imagination to understand the neural basis of episodic memory. Journal of Neuroscience, 27, 14365-14374. doi:10.1523/JNEUROSCI.454907.2007

Hintzman, D. L. (2011). Research strategy in the study of memory: Fads, fallacies, and the search for the "coordinates of truth.". Perspectives on Psychological Science, 6, 253-271.

Kahneman, D. (2011). Thinking: Fast and slow. New York, NY: Penguin.

Kelley, C., \& Jacoby, L. L. (2000). Recollection and familiarity: Processdissociation. In E. Tulving \& F. I. M. Craik (Eds.), The Oxford handbook of memory (pp. 215-228). Oxford, England: Oxford University Press.

Klein, S. B. (2013). The complex act of projecting oneself into the future. WIREs Cognitive Science, 4, 63-79. doi:10.1002/wcs.12.10

Kvavilashvili, L., \& Mandler, G. (2004). Out of one's mind: A study of involuntary semantic memories. Cognitive Psychology, 48, 47-94.

Kvavilashvili, L., \& Schlagman, S. (2011). Involuntary autobiographical memories in dysphoric mood: A laboratory study. Memory, 19, 331345. doi:10.1080/09658211.2011.568495

Mace, J. H. (2004). Involuntary autobiographical memories are highly dependent on abstract cuing: The Proustian view is incorrect. Applied Cognitive Psychology, 18, 893-899. doi:10.1002/acp.1020

Mace, J. H. (2006). Episodic remembering creates access to involuntary conscious memory: Demonstrating involuntary recall on a voluntary recall task. Memory, 14,917-924. doi:10.1080/ 09658210600759766

McMillan, R., Kaufman, S. B., \& Singer, J. L. (2013). Ode to positive constructive daydreaming. Frontiers in Perception Science, 4, 626.

Miles, A. N., \& Berntsen, D. (2011). Odour-induced mental time travel into the past and future: Do odour cues retain a unique link to our distant past? Memory, 19, 930-940.

Miles, A. N., \& Berntsen, D. (2015). The forgotten remindings: Personal remindings examined through self-probed retrospection during reading and writing. Cognition \& Consciousness, 33, 67-77.

Nisbett, R. E., \& Wilson, T. D. (1977). Telling more than we can know: Verbal reports on mental processes. Psychological Review, 84, 231259.

Rasmussen, A. S., \& Berntsen, D. (2013). The reality of the past versus the ideality of the future: Emotional valence and functional differences between past and future mental time travel. Memory \& Cognition, 41, 187-200.

Rasmussen, K. W., \& Berntsen, D. (2014). "I can see clearly now": The effect of cue imagability on mental time travel. Memory \& Cognition, 42, 1063-1075. doi:10.3758/s13421-014-0414-1

Ratcliff, R. (1993). Methods for dealing with reaction time outliers. Psychological Bulletin, 114, 510-532.

Rubin, D. (2014). Schema-driven construction of future autobiographical traumatic events: The future is much more troubling than the past. Journal of Experimental Psychology: General, 143, 612-630. doi: $10.1037 / \mathrm{a} 0032638$
Schacter, D. L. (2012). Adaptive constructive processes and the future of memory. American Psychologist, 67, 603-613. doi:10.1037/ a0029869

Schacter, D. L., \& Addis, D. R. (2007). The cognitive neuroscience of constructive memory: Remembering the past and imagining the future. Philosophical Transactions of the Royal Society of London. Series B: Biological Sciences, 362, 773-786.

Schacter, D. L., Addis, D. R., Hassabis, D., Martin, V. C., Spreng, R. N., \& Szpunar, K. K. (2012). The future of memory: Remembering, imagining and the brain. Neuron, 76, 677-694. doi:10.1016/j. neuron.2012.11.001

Schank, R. C. (1982). Dynamic memory. New York, NY: Cambridge University Press.

Schlagman, S., Kliegel, M., Schulz, J., \& Kvavilashvili, L. (2009). Differential effects of age on involuntary and voluntary autobiographical memory. Psychology and Aging, 24, 397-411. doi:10. 1037/a0015785

Schlagman, S., \& Kvavilashvili, L. (2008). Involuntary autobiographical memories in and outside the laboratory: How different are they from voluntary autobiographical memories? Memory \& Cognition, 36, 920-932.

Smallwood, J., Brown, K., Baird, B., \& Schooler, J. W. (2012). Cooperation between the default mode network and the frontalparietal network in the production of an internal train of thought. Brain Research, 1428, 60-70.

Smallwood, J., \& Schooler, J. W. (2006). The restless mind. Psychological Bulletin, 132, 946-958. doi:10.1037/0033-2909. 132.6.946

Spreng, R. N., \& Levine, B. (2006). The temporal distribution of past and future autobiographical events across the lifespan. Memory \& Cognition, 34, 1644-1651.

Ste-Marie, D. M., \& Jacoby, L. L. (1993). Spontaneous versus directed recognition: The relativity of automaticity. Journal of Experimental Psychology: Learning, Memory, and Cognition, 19, 777-788.

Szpunar, K. K. (2010). Evidence for an implicit influence of memory on future thinking. Memory \& Cognition, 38, 531-540. doi:10.3758/ MC.38.5.531

Szpunar, K. K., Watson, J. M., \& McDermott, K. B. (2007). Neural substrates of envisioning the future. Proceedings of the National Academy of Sciences of the USA, 104, 642-647.

Tulving, E. (2002). Episodic memory: From mind to brain. Annual Review of Psychology, 53, 1-25.

Uzer, T., Lee, P. J., \& Brown, N. R. (2012). On the prevalence of directly retrieved autobiographical memories. Journal of Experimental Psychology: Learning, Memory \& Cognition, 38, 1296-1308. doi: 10.1037/a0028142

Vannucci, M., Batool, I., Pelagatti, C., \& Mazzoni, G. (2014). Modifying the frequency and characteristics of involuntary autobiographical memories. PLOS ONE, 9(4), e89582. doi:10.1371/journal.pone. 0089582

Wheeler, M., Stuss, D., \& Tulving, E. (1997). Toward a theory of episodic memory: The frontal lobes and autonoetic consciousness. Psychological Bulletin, 121, 331-354. 